\title{
A comparative study of two reconstructive methods and different recommendations in intracavitary brachytherapy
}

\author{
NVN Madhusudhana Sresty, MSc, Dip.R.P., PhD', Thogata Ramanjappa, MSc, PhD², C. Ramakrishna Rao, MSc, Dip.R.P.․, \\ A Krishnam Raju, MD², KR Muralidhar, MSc, Dip.R.P., M.Phill, PhD' \\ 'Department of Radiation Physics, Basavatarakam Indo-American Cancer Hospital \& Research Institute, Hyderabad, India, \\ 2Department of Physics, Sri Krishnadevaraya University. Anantapur, India, ${ }^{3}$ Department of Radiation Physics, MNJ Institute of Oncology \& RCC, \\ Hyderabad, India, ${ }^{2}$ Department of Radiotherapy, Basavatarakam Indo-American Cancer Hospital \& Research Institute, Hyderabad, India
}

\begin{abstract}
Purpose: Intracavitary brachytherapy (ICB) is a widely used technique in the treatment of cervical cancer. In our Institute, we use different reconstructive methods in the conventional planning procedure. The main aim of this study was to compare these methods using critical organ doses obtained in various treatment plans. There is a small difference in the recommendations in selecting bladder dose point between ICRU (International Commission on Radiation Units \& Measurements) -38 and ABS (American Brachytherapy Society). The second objective of the study was to find the difference in bladder dose using both recommendations.

Material and methods: We have selected two methods: variable angle method (M1) and orthogonal method (M2). Two orthogonal sets of radiographs were taken into consideration using conventional simulator. All four radiographs were used in M1 and only two radiographs were used in M2. Bladder and rectum doses were calculated using ICRU-38 recommendations. For maximum bladder dose reference point as per the ABS recommendation, 4 to 5 reference points were marked on Foley's balloon.

Results: $64 \%$ of plans were showing more bladder dose and $50 \%$ of plans presented more rectum dose in M1 compared to M2. Many of the plans reviled maximum bladder dose point, other than ICRU-38 bladder point in both methods. Variation was exceeded in 5\% of considerable number of plans.

Conclusions: We observed a difference in critical organ dose between two studied methods. There is an advantage of using variable angle reconstruction method in identifying the catheters. It is useful to follow ABS recommendation to find maximum bladder dose.
\end{abstract}

Key words: HDR, cervical cancer, ICRU, ABS, reconstruction.

\section{Purpose}

Brachytherapy plays a significant role in the treatment of many cancers. High dose rate (HDR) brachytherapy with remote after loading system using Ir-192 radioisotope became the major treatment modality for gynaecological cancers. In some advanced cases where surgery is not possible, brachytherapy is very useful [1-3]. During HDR treatment session, single source moves from machine safe position to different pre-planned dwell positions [4]. After the completion of all dwell locations, the source returns to a safe. The main advantage with HDR brachytherapy is the miniature size radioactive source giving optimised radiation dose distributions by variable dwell times. In our Institute most of the gynaecological malignancies are treated with the use of external beam radiotherapy (EBRT) and intracavitary brachytherapy (ICB). All cases are treated after computerized treatment planning. Although, more modern approaches were recently presented in many papers, such as image guided, CT or MR supported brachytherapy, along with new recommendations for critical organs other than point dose measurements. However, in developing countries, most of the institutions follow only conventional planning. In this conservative brachytherapy planning, dosimetry of ICB is carried out using orthogonal radiographs, where point doses to critical structures are calculated [5]. For the reconstruction of source position localization, different techniques are available in the treatment planning system. We have chosen two prime reconstructive methods.

a) Variable angle reconstructive method (M1): A total of four images are taken in this method, including two 
orthogonal sets. The gantry angles of one set of films is selected in a way that both localisations of dummies in the tandem and ovoid's could be seen without any obstruction. Therefore, the applicator could easily be marked for the reconstruction of source positions. Critical organ reference points are marked using one more set of orthogonal films such as antero-posterior (AP) and lateral films.

b) Orthogonal reconstructive method (M2): This is the most common method used in intracavitary brachytherapy [6]. Antero-posterior (AP) and lateral orthogonal films of the intracavitary implant are taken. Catheters reconstruction and critical organ reference points are marked using two films only. The main disadvantage of this method is the difficulty in distinguishing the reconstruction of catheters and dummies in the lateral film due to thickness of tissue and overlap of the catheters in actual orthogonal radiograph images.

The main purpose of this study was to compare these two methods based on doses to the critical organs. The rectum dose reference point as per ABS (American Brachytherapy Society) and ICRU (International Commission on Radiation Units and Measurements) in 38 guidelines is the same. However, in order to localize bladder point, ABS uses ICRU 38 [7] bladder point with a small change. Therefore, we intended to study the variation between two recommended points also.

\section{Material and methods}

We compared 50 plans of 28 patients with FIGO stage IIB, IIIA and IIIB of cervical cancer. All patients received EBRT dose of $50 \mathrm{~Gy}$ in 25 fractions. Brachytherapy started during the course of EBRT after 30 Gy. HDR-ICB was planned $21 \mathrm{~Gy}$ in three fractions with one week interval. Three channel Fletcher Suit applicator was used. ICB procedure consisted of placement of intrauterine tandem into the uterine cavity and the ovoid's in the vagina at the level of fornices. A Foley's catheter was inserted and balloon was filled with $7 \mathrm{cc}$ urograffin to identify the bladder. Radio opaque gauge was packed in anterior and posterior vaginal space to move the organs (bladder and rectum) away from the applicator. Radiographs for both methods were taken by using conventional simulator. In lateral radiograph, the bladder reference point was taken on the posterior surface of the balloon, along with the antero-posterior line drawn through its centre (as per ICRU-38). ABS recommends that the reference point should correspond with the maximum dose. Therefore, to know the maximum bladder dose point, 4 to 5 reference points were marked on the posterior surface of the balloon apart from ICRU reference point. On AP radiograph, corresponding bladder reference points based on lateral radiograph were marked on a vertical line through the centre of the balloon. For rectum reference point on lateral radiograph, anteroposterior line was drawn from the lower end of the intrauterine source. Reference point was taken on this line $5 \mathrm{~mm}$ behind posterior vaginal wall. On AP radiograph rectum reference point was at the lower end of the intrauterine source. A dose of 7 Gy was prescribed to point A, which was positioned by measuring $2 \mathrm{~cm}$ above the lower end of the intrauterine tube and $2 \mathrm{~cm}$ lateral to the uterine sources.

Our Institute is equipped with MicroSelectron HDR (Nucletron ${ }^{\circledR}$, Veneendal, the Netherlands) Brachytherapy unit, PLATO 3D TPS (PLATO Sunrise Maintenance Release 2, Nucletron ${ }^{\circledR}$ ) and XIMATRON (Crawley ${ }^{\circledR}$, UK) RT simulator. We use DICOM/SC ${ }^{\circledR}$ facility between simulator, treatment planning system and control console of HDR unit. Among M1 and M2, we usually follow M1 because of its advantage for the user in the planning procedure.

In $\mathrm{M} 1$, four simulated images with different angles $0^{\circ}$, $45^{\circ}, 90^{\circ}$ and $315^{\circ}$ were used. Catheter reconstruction was done using radiographs of the gantry angles $45^{\circ}$ and $315^{\circ}$. Critical organ reference points were marked using $0^{\circ}$ and $90^{\circ}$ radiographs. In M2 catheter reconstruction and reference points were identified by using 00 and 900 radiographs only. Bladder reference point was identified by using both ICRU-38 and ABS. Rectum reference point is the same in both recommendations. Source positions were loaded according to the standard loading pattern based on Manchester system. Identical step size $(5 \mathrm{~mm})$ was applied in both methods. Treatment planning was completed in TPS and bladder and rectum doses were calculated. Isodose distribution is generated in the planning system by defining and specifying the dose to point $\mathrm{A}$ in both methods. Treatment was given using MicroSelectron HDR unit.

\section{Results}

Figures 1 and 2 represent bladder and rectum doses in M1 and M2, respectively. Both reconstruction methods were shown as different dots. Vertical line between the dots shows the difference of doses among the methods. Observed doses were expressed in percentages with respect to point A dose $(7 \mathrm{~Gy}=100 \%)$.

\section{Bladder dose variation}

Observed doses were more in 32 plans and less in 13 plans in M1 when compared to M2, respectively, and equal doses in 5 plans. In 17 plans, dissimilarity occurred in more than $5 \%$. Maximum variation observed was in $8.2 \%$ of all plans (Fig. 1). Average difference between M1 and M2 for all the data was $2.9 \%$.

\section{Rectum dose variation}

Observed doses were more in 25 plans and less in 21 plans in M1 when compared to M2, and equal doses in 4 plans. In 10 plans, variation was exceeded in 5\%. Maximum variation was found in $6.6 \%$ (Fig. 2). Average difference between $\mathrm{M} 1$ and $\mathrm{M} 2$ for all the data was $2.1 \%$.

\section{ICRU 38 and ABS in bladder point specification}

Table 1 shows the bladder doses according to ICRU 38 and ABS recommendations in M1 and M2, respectively. We observed that, in majority of cases, there was some other bladder point which showed maximum dose other than ICRU 38 bladder points in both M1 and M2. ABS recommends taking the point corresponding to the maximum dose on the surface of the Foley's balloon. Twenty eight 


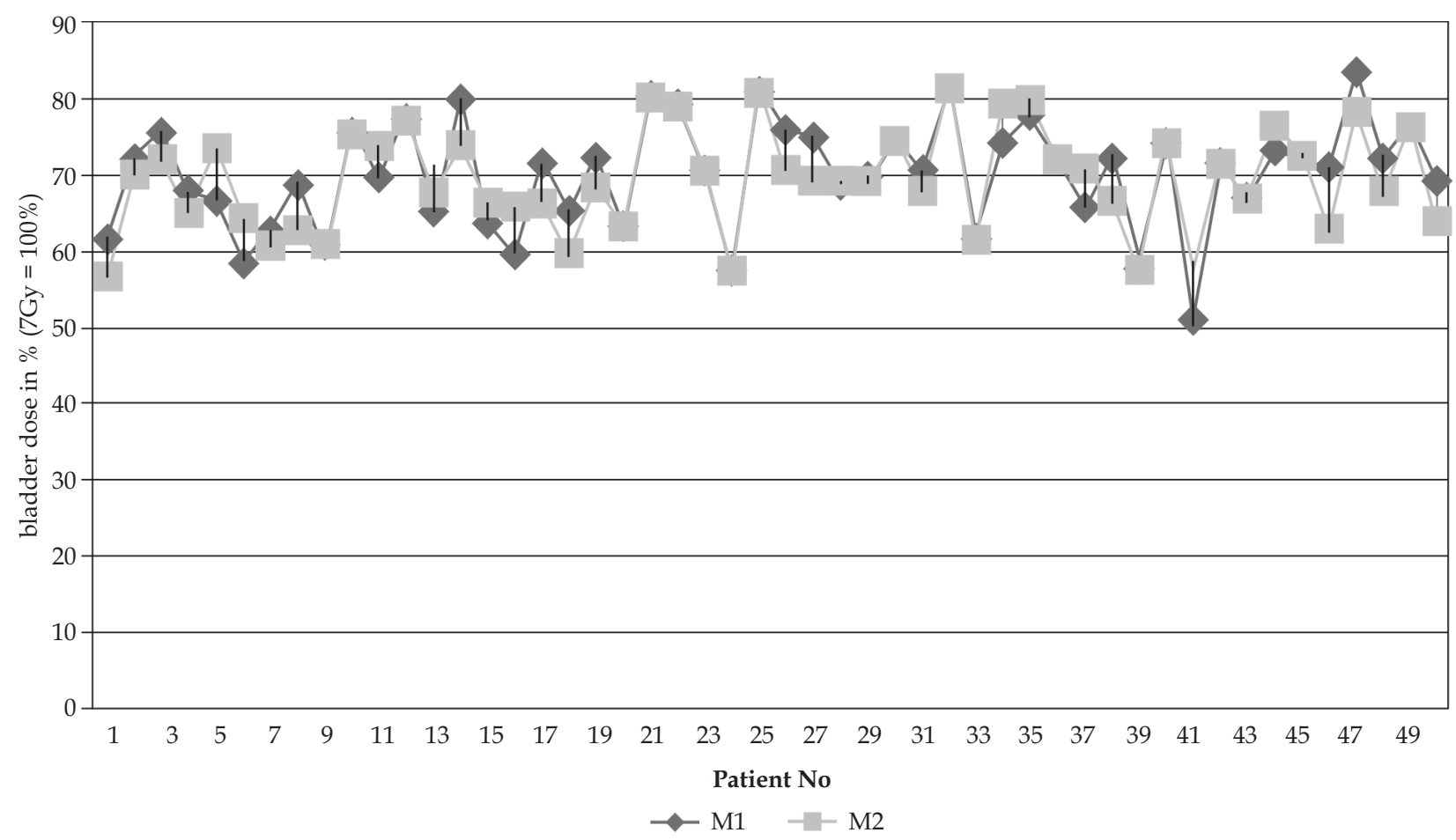

Fig. 1. Bladder doses expressed in percentage $(7 \mathrm{~Gy}=100 \%)$ in variable angle reconstructive method (M1) and orthogonal reconstruction method (M2) presented as different dots in this line graph. Vertical lines between the dots represent the difference in bladder doses for each patient

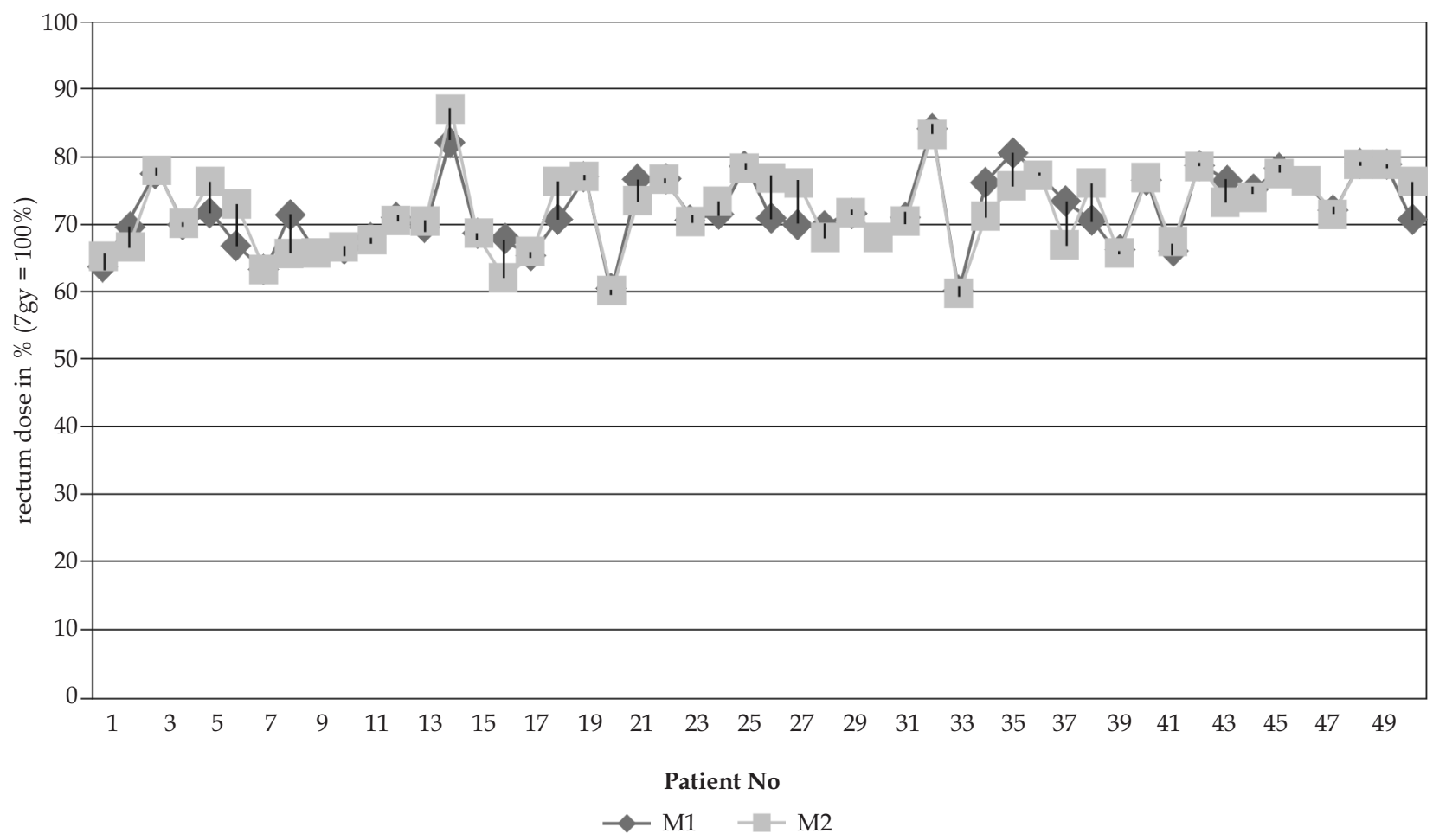

Fig. 2. Rectum doses expressed in percentage $(7 \mathrm{~Gy}=100 \%)$ in variable angle reconstructive method (M1) and orthogonal reconstruction method (M2) presented as different dots in this line graph. Vertical lines between the dots represent the difference in rectum doses for each patient 
Table 1. Bladder dose expressed in percentage (7 Gy $=100 \%)$ at reference point based on ICRU-38 and ABS recommendations in $\mathrm{M} 1$ and $M 2$

\begin{tabular}{|c|c|c|c|c|}
\hline S.NO & $\begin{array}{c}\text { ICRU } \\
\text { Bladder } \\
\text { point (M1) }\end{array}$ & $\begin{array}{c}\text { ABS } \\
\text { Bladder } \\
\text { point (M1) }\end{array}$ & $\begin{array}{c}\text { ICRU } \\
\text { Bladder } \\
\text { point (M2) }\end{array}$ & $\begin{array}{c}\text { ABS } \\
\text { Bladder } \\
\text { point (M2) }\end{array}$ \\
\hline 1 & 61.7 & 65.2 & 56.5 & 59.4 \\
\hline 2 & 72.4 & 73.5 & 70.1 & 71.6 \\
\hline 3 & 75.8 & 75.8 & 72.1 & 73.2 \\
\hline 4 & 67.9 & 69.4 & 65 & 66.8 \\
\hline 5 & 66.8 & 66.8 & 73.5 & 73.5 \\
\hline 6 & 58.5 & 60.1 & 64.3 & 66.3 \\
\hline 7 & 62.8 & 62.8 & 60.7 & 60.7 \\
\hline 8 & 68.8 & 68.8 & 62.7 & 65.2 \\
\hline 9 & 60.8 & 68.8 & 60.8 & 67.9 \\
\hline 10 & 75.9 & 75.9 & 75.3 & 75.3 \\
\hline 11 & 69.8 & 73.7 & 73.8 & 75.8 \\
\hline 12 & 77.9 & 77.9 & 77.2 & 77.2 \\
\hline 13 & 65.3 & 65.3 & 67.7 & 67.7 \\
\hline 14 & 80.1 & 80.1 & 73.9 & 73.9 \\
\hline 15 & 63.7 & 68.6 & 66.4 & 69.3 \\
\hline 16 & 59.7 & 64.7 & 65.8 & 65.8 \\
\hline 17 & 71.7 & 74.9 & 66.3 & 70.1 \\
\hline 18 & 65.5 & 65.5 & 59.7 & 62.2 \\
\hline 19 & 72.4 & 72.4 & 68.3 & 68.3 \\
\hline 20 & 63.5 & 66.4 & 63.2 & 68.4 \\
\hline 21 & 80.5 & 80.5 & 80.3 & 80.3 \\
\hline 22 & 79.5 & 80.8 & 79.1 & 80.4 \\
\hline 23 & 70.6 & 71.5 & 70.6 & 74.5 \\
\hline 24 & 57.7 & 57.7 & 57.3 & 57.3 \\
\hline 25 & 81.2 & 82.2 & 80.9 & 81.9 \\
\hline 26 & 76 & 77.7 & 70.7 & 73.2 \\
\hline 27 & 75.1 & 75.1 & 69.3 & 69.3 \\
\hline 28 & 68.8 & 73.9 & 69.2 & 70.5 \\
\hline 29 & 69.9 & 70.9 & 69.1 & 71.1 \\
\hline 30 & 74.5 & 76.7 & 74.5 & 76.9 \\
\hline 31 & 70.8 & 70.8 & 67.9 & 67.9 \\
\hline 32 & 81.6 & 81.6 & 81.5 & 81.5 \\
\hline 33 & 62.5 & 68.9 & 61.4 & 66.8 \\
\hline 34 & 74.4 & 76.5 & 79.5 & 81.7 \\
\hline 35 & 77.8 & 79.2 & 79.9 & 81.5 \\
\hline 36 & 71.7 & 71.7 & 72.1 & 72.1 \\
\hline 37 & 65.8 & 67.5 & 70.8 & 73.9 \\
\hline 38 & 72.4 & 72.4 & 66.7 & 70.7 \\
\hline 39 & 59.6 & 65.7 & 57.5 & 61.2 \\
\hline 40 & 74.2 & 74.2 & 74.2 & 74.2 \\
\hline 41 & 51.3 & 55.8 & 57.8 & 61.4 \\
\hline 42 & 71.4 & 71.4 & 71.4 & 71.4 \\
\hline 43 & 67.5 & 68.9 & 66.8 & 68.2 \\
\hline 44 & 73.4 & 74.4 & 76.5 & 78.8 \\
\hline 45 & 72.9 & 72.9 & 72.5 & 72.5 \\
\hline 46 & 71.1 & 72.7 & 62.9 & 68.7 \\
\hline 47 & 83.6 & 83.6 & 78.3 & 78.3 \\
\hline 48 & 72.4 & 74.8 & 67.9 & 69.3 \\
\hline 49 & 76.6 & 78.3 & 76.2 & 79.5 \\
\hline 50 & 69.4 & 69.4 & 63.8 & 63.8 \\
\hline
\end{tabular}

plans in M1 and 31 plans in M2 had a different bladder point which was equal to maximum dose (Table 1).

\section{Discussion}

It was observed that the same critical organ doses in both methods were found in very few plans. In general, doses to the critical organs were more, equal or less depending on the reconstruction pattern of the implant. In this study, we observed that, M1 showed more bladder dose in $64 \%$ (32 out of 50) of the plans and more rectum dose in 50\% (25 out of 50) of the plans compared to M2 due to the change in reconstruction of the catheters only. In the same way, M1 showed less doses compared with M2 for remaining patients for the same reason. Though, the variation occurred in less than 5\% for most of the plans, it was exceeded in $5 \%$ for considerable number of plans.

In variable angle method, we could identify the tandem and ovoid's separately by identification of dummies, and the reconstruction was completed with oblique orthogonal radiographs. Nevertheless, in case of orthogonal method, clarity was not possible to obtain due to overlapping of ovoid's in lateral radiograph. Secondly, dummies were difficult to identify in lateral radiograph because of thickness of tissues. Because of the above difficulties, the reconstruction of the applicator and distribution of doses were different in two selected methods.

\section{Conclusions}

In this comparative study of M1 and M2 for critical organ doses we have observed a difference in doses to critical organ. In M1, the reconstruction procedure is more reliable because of its advantage in identifying the exact position of the applicator and the orientation of changing the angle at the time of simulation. We concluded that calculated doses were accurate in M1 when compared with M2 in plans of selected patients. Therefore, M1 is preferable choice for ICB planning. We have observed a different bladder point which corresponds to maximum dose other than ICRU 38 points in majority of the cases in both methods. From the result, we concluded that, in order to identify the maximum dose to a bladder, it is recommended to mark more points on the posterior surface of Foley's balloon apart from ICRU 38 points. We also observed variation in doses in more than $5 \%$ of plans which is a significant outcome that should be considered in treatment planning.

\section{Acknowledgement}

We wish to thank Mr. Uday Kumar, Statistical Officer of our department for contributing in statistical analysis.

\section{References}

1. Nag S, Erickson B, Thomadsen B et al. The American Brachytherapy Society recommendations for high-dose-rate brachytherapy for carcinoma of the cervix. Int J Radiat Oncol Biol Phys 2000; 48: 201-211.

2. Foroudi F, Bull CA, Gebski V. Radiation therapy for cervix carcinoma: benefits of individualized dosimetry. Clin Oncol (R Coll Radiol) 2002; 14: 43-49. 
3. Sunder S, Symonds P, Deehan C. Tolerance of pelvic organs to radiation treatment for carcinoma of cervix. Clin Oncol ( $R$ Coll Radiol) 2003; 15: 240-247.

4. Henschke UK, Hilaris BS, Mahan GD. Remote after loading with intracavitary applicators. Radiology 1964; 83: 344-345.

5. Jamema S, Sherly S, Umesh M et al. Dosimetric evaluation of rectum and bladder using image-based CT planning and orthogonal radiographs with ICRU 38 recommendations in intracavitary brachytherapy. JMP 2008; 33: 3-8.

6. Joslin CA, Flynn A, Hall EJ. Principles and practice of brachytherapy. Arnold, London 2001.

7. International commission on radiation units and measurements (ICRU) report 38: Dose volume specification for reporting intracavitary brachytherapy in genecology. ICRU, Bethesda 1985. 